\title{
Diagnostic Value of $\alpha$-Glutathione S-transferase in Acute Mesenteric Ischemia
}

\author{
Ahmed Abdel Fattah Elshoura ${ }^{1}$, Hala Desouky Ibrahim ${ }^{2}$, Sahar Mohey Hazzaa ${ }^{3}$, \\ Ola Abdel Fattah Elshora ${ }^{3}$, Gamal Ibrahim Moussa ${ }^{1}$ \\ ${ }^{1}$ General Surgery Department, Faculty of Medicine, Tanta University, Tanta, Egypt \\ ${ }^{2}$ Emergency \& Traumatology Department, Faculty of Medicine, Tanta University, Tanta, Egypt \\ ${ }^{3}$ Clinical Pathology Department, Faculty of Medicine, Tanta University, Tanta, Egypt
}

\section{Email address:}

Ahmed.elshoura@med.tanta.edu.eg (A. A. F. Elshoura), ahmed.elshora28@hotmail.com (A. A. F. Elshoura)

\section{To cite this article:}

Ahmed Abdel Fattah Elshoura, Hala Desouky Ibrahim, Sahar Mohey Hazzaa, Ola Abdel Fattah Elshora, Gamal Ibrahim Moussa. Diagnostic Value of $\alpha$-Glutathione S-transferase in Acute Mesenteric Ischemia. Journal of Surgery. Vol. 6, No. 4, 2018, pp. 107-111. doi: $10.11648 /$ j.js.20180604.16

Received: July 10, 2018; Accepted: July 20, 2018; Published: August 14, 2018

\begin{abstract}
Background: Acute mesenteric ischemia (AMI) is a life-threatening problem, and the early clinical manifestations of it are non-specific. Despite the advances in laboratory \& radiological diagnostic procedures, the mortality rate of AMI is still high. Aim: The purpose of the study to evaluate the ability of $\alpha$-glutathione S-transferase ( $\alpha$-GST) to predict AMI in patients with abdominal pain. Methods: This prospective study was conducted in Tanta University Hospitals between November 2014 and December 2017. Nighty patients were included after clinical suspicious of AMI. Serum levels of $\alpha$ - GST were measured in the collected stored samples, and other biochemical markers were performed (e.g. LDH, PH, and WBC). AMI was confirmed by CT angiography or laparotomy. Patients without ischemic bowel were considered to be controls. Results: A total of 90 patients in the study, the ischemic group included 52 patients, and the non-ischemic group included 38 patients. Patients with intestinal ischemia had significant higher serum values of D-dimer, LDH and $\alpha-$ GST. Where $\alpha$-GST diagnosed AMI by accuracy of $84.4 \%$. Conclusion: Measurement of plasma $\alpha$-GST is a simple, early, easy, and effective procedure making it a useful serum biomarker for early diagnosis of AMI.
\end{abstract}

Keywords: Acute Abdomen, Mesenteric Ischemia, $\alpha$-glutathione S-transferase

\section{Introduction}

Acute mesenteric ischemia (AMI) is a life-threatening surgical \& vascular emergency characterized by the clinical triad of pain disproportionate to clinical features, diarrhea progressing to obstipation, and a probable source of thromboembolism. Despite the advances in laboratory \& radiological diagnostic procedures, the mortality rate of AMI is up to $80 \%$ with failure of prompt diagnosis is a considerable contributing factor in mortality [1].

The early clinical manifestations of AMI are non-specific. Also, laboratory data and imaging methods including duplex ultrasonography and computerized tomographic angiography (CTA) are not sufficiently sensitive in diagnosis of AMI [2].

Although CTA is considered the gold standard for diagnosis, as it detects the site of vascular occlusion, allows proper differentiation between occlusive \& non-occlusive causes of AMI \& may provide other diagnoses in suspected cases, yet mesenteric angiography might be difficult to access, often underutilized in the emergency \& critical settings where the logistics of performing such radiological investigation for a ventilated patient could be a great challenge [3]. It is unlikely whether all AMI characteristics are evaluated by specialized radiologists at all practice locations [4].

Therefore, the need for a new diagnostic marker is essential as earlier diagnosis with a simple laboratory test would be a major breakthrough. An ideal biochemical marker for AMI should be specific, sensitive, released from the intestinal mucosa to detect ischemia at the earliest stage, noninvasive and measurable in the peripheral blood [5].

The GST superfamily function to combat intracellular 
stress and maintain cellular hemostasis. GST catalyzes the conjugation of glutathione to a number of toxic substrates known to be elevated in oxidative stress, making them more water soluble and facilitating fecal or urinary excretion. The alpha subunit of (GST) in humans has been shown to have activity I the colon, small bowel and liver. For these reasons alpha glutathione transferase (GST) activity may be a useful predictor of AMI [6].

This study was designed to evaluate the ability of $\alpha$ glutathione S-transferase (GST) to predict AMI in patients with abdominal pain with suggestive clinical features.

\section{Patients \& Methods}

This prospective study was conducted in Tanta University Hospitals between November 2014 and December 2017. Nighty patients were included after clinical suspicious of AMI. Thorough history, physical examination and investigations including radiological and routine laboratory studies were carried out for all patients. Written informed consents were taken from all patients when possible or their first-degree relatives. This study was approved by the ethical committee of Tanta University Hospitals.

The inclusion criteria included abdominal pain or distention, at least one mesenteric vessel obstruction confirmed by CTA, digital subtraction angiography, or laparotomy exploration and intestinal wall injury at CT scan.

Patients with history of malignancy, chronic hematological diseases, irreversible acute hepatic or renal failure, and cranial trauma were excluded.

The control group included patients who presented to the emergency department with abdominal pain confirmed by imaging or laparotomy to be due to causes other than AMI.

\subsection{Collection of Blood Samples}

A blood sample $(2.5 \mathrm{ml})$ was collected from every patient and preserved in an ethylene diamine tetra-acetic acid (EDTA)-coated standard phlebotomy tube. In Clinical Pathology Department of Tanta University Hospitals, the plasma was immediately separated and stored at $\left(-20^{\circ} \mathrm{C}\right)$. Plasma levels of $\alpha$ - GST were measured in the collected stored samples using a commercially available enzyme linked radio-immunosorbent assay (ELISA, Biogene. A. Company, Shanghai).

According to Delaney et al., 1999 [7], $\alpha$-GST serum level $\geq 4 \mathrm{ng} / \mathrm{ml}$ was considered elevated. Other biochemical investigations that were performed at the time of initial evaluation included serum $\mathrm{LDH}, \mathrm{PH}$, white blood cell count (WBC), amylase, C-reactive protein (CRP), D-dimer, alanine transferase (ALT) and aspartate transferase (AST). AMI was confirmed by $\mathrm{CT}$ angiography or laparotomy. Patients without ischemic bowel were considered to be controls, while those with ischemic bowel were placed in the AMI group.

\subsection{Statistical Analysis}

Statistical analysis was performed using SPSS IBM software program v21. Numerical data was presented as mean and standard deviation. Categorical data was presented as number and percentage distribution. Independent t-test was used as a test of significance for comparison between ischemic and non-ischemic group. Level of significance was adopted at $\mathrm{p}$-value less than 0.05 .

\section{Results}

A total of 90 patients in our study with acute abdominal pathology were divided into 2 groups according to presence or absence of AMI: ischemic and non-ischemic (control) groups. The ischemic group included 52 patients, with 36 males $(69.2 \%)$ and 16 females $(30.8 \%)$, with mean age of 55.6 (28-85) years. The non-ischemic group included 38 patients with 22 males (57.9\%), and 16 females (42.1\%) with mean age of $43(20-65)$ years. In the ischemic group, all patients had occlusive AMI, 42 cases $(80.8 \%)$ had small bowel mesenteric ischemia, 2 cases $(3.8 \%)$ had colonic ischemia and 8 cases $(15.4 \%)$ had both small bowel and colonic ischemia.

The diagnoses in the control group were as follow: perforated peptic ulcer in 6 cases $(15.8 \%)$, perforated appendix in 8 cases $(21.1 \%)$, diverticulitis in 4 cases (10.5\%), salpengeo-oophritis in 4 cases $(10.5 \%), 8$ cases with pancreatitis $(21.1 \%), 6$ cases with intestinal obstruction (15.8\%) and 2 cases with pyonephrosis (5.2\%).

Acute mesenteric ischemia was confirmed angiograpphically in 41 cases $(78.9 \%)$, and at laparotomy in 11 cases $(21.1 \%)$.

In ischemic group, 19 patients treated medically by anticoagulation $(36.5 \%)$, while surgical intervention was done in 33 cases $(63.5 \%) .2^{\text {nd }}$ laparotomy was needed in 14 cases $(42.4 \%)$. The overall 30 -day mortality was 21 cases (40.4\%).

Patients with intestinal ischemia had significant higher serum values of D-dimer, LDH and $\alpha$-GST than patients without intestinal ischemia as shown in Table 1.

Table 1. Plasma biomarkers in patients with and without intestinal ischemia.

\begin{tabular}{llll}
\hline \multirow{2}{*}{ Parameter } & $\begin{array}{l}\text { Non-ischemic } \\
\text { Mean } \pm \text { SD (range) }\end{array}$ & $\begin{array}{l}\text { Ischemic } \\
\text { Mean } \pm \text { SD (range) }\end{array}$ & $\begin{array}{l}\text { t-test } \\
\text { p-value }\end{array}$ \\
\hline \multirow{2}{*}{ Amylase } & $234.5 \pm 508.7$ & $73.6 \pm 65.5$ & 0.31 \\
& $(15-2000)$ & $(22-234)$ & 0.75 \\
\multirow{2}{*}{$\alpha$-GST } & $2.5 \pm 5.3$ & $14.96 \pm 8.2$ & 8.2 \\
& $(0.6-12.1)$ & $(0.9-45.5)$ & $<0.001^{*}$ \\
CRP & $115.4 \pm 95.5$ & $183.5 \pm 135.4$ & 1.79 \\
& $(12-340)$ & $(36-530)$ & 0.079 \\
D-dimer & $508.2 \pm 733.9$ & $1009.3 \pm 926.9$ & 2.78 \\
& $(77-2500)$ & $(90-3040)$ & $0.005^{*}$ \\
LDH & $289.4 \pm 215.1$ & $556.4 \pm 666.2$ & 2.4 \\
& $(50-100)$ & $(126-3568)$ & $0.016^{*}$ \\
PH & $7.4 \pm 0.1$ & $7.4 \pm 0.1$ & 1.36 \\
& $(7.23-7.5)$ & $(7.3-7.6)$ & 0.1 \\
WBCs & $15581.6 \pm 9400.6$ & $13038 \pm 6167.1$ & 0.37 \\
& $(4600-36000)$ & $(4700-30000)$ & 0.7 \\
\hline
\end{tabular}


Alpha GST was elevated in 46 patients of the ischemic group $(88.5 \%)$ and was normal in 6 patients only $(11.5 \%)$. Its mean plasma value was $14.96 \mathrm{ng} / \mathrm{ml}(0.9-45.5 \mathrm{ng} / \mathrm{ml})$. In non-ischemic group $\alpha$-GST increased only in 8 patients $(21.1 \%)$, was normal in 30 patients $(78.9 \%)$ and ranged from 0.6 to $12.1 \mathrm{ng} / \mathrm{ml}$ with a mean $2.5 \mathrm{ng} / \mathrm{ml}$. $\alpha$-GST was more accurate than other clinical parameters (WBC, LDH, DDimmer, amylase, and PH as shown in Table 2.
When using the cut-off value $4 \mathrm{ng} / \mathrm{ml}$, the diagnostic properties of $\alpha$-GST in patients with intestinal ischaemia were as following: sensitivity $88.4 \%$, specificity $78.9 \%$, and overall accuracy $84.4 \%$. The corresponding figures for Ddimer Sensitivity $76.9 \%$, specificity $57.9 \%$, and overall accuracy $68.9 \%$. Also LDH Sensitivity $82.6 \%$, specificity $26.3 \%$, and overall accuracy $58.8 \%$ as shown in Table 2 .

Table 2. Diagnostic properties of plasma biomarkers for intestinal ischemia.

\begin{tabular}{|c|c|c|c|c|c|c|c|c|c|c|}
\hline Parameters & Normal ranges & TP & FP & $\mathbf{F N}$ & TN & Sensitivity & Specificity & PPV & NPV & Accuracy \\
\hline WBCs & $4000-11000$ & 32 & 22 & 20 & 16 & 65.4 & 42.1 & 60.7 & 47.1 & 55.6 \\
\hline LDH & $95-195$ & 43 & 28 & 9 & 10 & 82.6 & 26.3 & 60.5 & 52.6 & 58.8 \\
\hline D-dimer & $\leqslant 250$ & 40 & 16 & 12 & 22 & 76.9 & 57.9 & 71.4 & 64.7 & 68.9 \\
\hline Amylase & $23-85$ & 12 & 6 & 40 & 32 & 23.1 & 84.2 & 66.7 & 44.4 & 48.9 \\
\hline $\mathrm{PH}$ & $7.35-7.45$ & 30 & 18 & 22 & 20 & 57.7 & 52.6 & 62.5 & 47.6 & 55.6 \\
\hline$\alpha-\mathrm{GST}$ & $\leqslant 4$ & 46 & 8 & 6 & 30 & 88.4 & 78.9 & 85.1 & 83.3 & 84.4 \\
\hline
\end{tabular}

$\mathrm{TP}=$ True positive $\mathrm{FP}=$ False Positive $\mathrm{FN}=$ False Negative $\mathrm{TN}=$ True Negative $\mathrm{PPV}=$ Positive Predictive value NPV= Negative Predictive Value

\section{Discussion}

Acute intestinal ischemia still has significantly high morbidity and mortality rates in the last decades, most probably due to delayed diagnosis \& paucity of therapeutic options. The delay in the diagnosis is attributed to nonspecific clinical features at presentation \& the lack of specific serum markers for rapid diagnosis [8].

Intestinal ischemia is a broad entity that may be attributed to physiological conditions (e.g. exercise, stress) and to pathophysiological events (e.g. acute mesenteric ischemia, aortic surgery). This may in part, illustrates the difficulty to obtain useful diagnostic markers [9].

The gold standard for AMI diagnosis remains thin slice CT and CTA. These radiological tools can easily rule out most of the other etiologies of acute abdomen, detects the site $\&$ the type of intestinal wall ischemia. However, these radiological modalities could be difficult to manage in emergency situations [10].

Although there are multiple laboratory markers used in clinical practice in the setting of suspected AMI (e.g. lactate, LDH, WBCs \& CRP), none of them has good diagnostic accuracy \& they can't differentiate between ischemic \& nonischemic patients [2]. But they may reflect disease progression and should not be used for diagnostic purposes [11].

In our study, small bowel ischemia was found in 42 patients $(80.08 \%)$, colonic ischemia was found in 2 patients $(3.8 \%)$ and both small bowel and colonic affection were present in 8 patients $(15.4 \%)$, which match the results of Yusuf et al, 2016 where (72.4\%) patients exhibited only small bowel involvement, but (27.6\%) exhibited both small intestinal and colonic involvement [5].

However, Gearhart et al, 2003 found small bowel ischemia in $40 \%$ of patients, colonic ischemia in $49 \%$ of patients \& $11 \%$ of patients had both small and colonic ischemia [12].

In the study of Delaney et al, $1999,50 \%$ of patients with AMI had occlusive small bowel mesenteric ischemia \& 50\% of patients had non-occlusive mesenteric ischemia affecting both small and colonic bowels [7].

In our study, the overall 30-day mortality in AMI group was 21 cases $(40.4 \%)$. This copes with the results of Yusuf et al, 2016who reported 30-day mortality rate of $51.7 \%$ in AMI patients [5].

However, our results were higher than those reported by Duran et al., 2015 \& Shuofei et al., 2014 (30.8\% \& 12\% respectively) [13], [14].

It is known that serum levels of laboratory biomarkers are the difference between the rate of production of those biomarkers from the affected tissues \& the rate of their elimination (mostly by the kidney or the liver) or elimination by pathological losses (as excessive secretion over a barrier e.g. peritoneum, mucous membranes). Therefore, increased serum biomarker level may be due to increased production or decreased elimination. The optimal time window to detect $\&$ measure a serum biomarker may differ depending on which tissue is producing it and on the mechanism by which biomarker exits from the cells into the blood stream. In AMI, the cause (thrombus, embolus or hypo-perfusion due to shock) and severity of tissue damage could affect the dynamics of biomarkers production \& elimination [15].

In our study, there is significant difference between the ischemic and non-ischemic groups of patients only in $\mathrm{LDH}$, D- dimer, and $\alpha$-GST.

In terms of AMI diagnosis, our study showed that the sensitivity and specificity of WBC, amylase, $\mathrm{PH}$ and LDH were $(65.4 \% \& 42.1 \%),(23.1 \% \& 84.2 \%),(57.7 \% \& 52.6 \%)$ and $(82.6 \% \& 26.3 \%)$ respectively.

In an earlier study, WBC for AMI diagnosis showed sensitivity, and specificity, $86.21 \%$, and $95.14 \%$, respectively [5].

Gearhart et al, 2003 reported sensitivities, specificities, and accuracy for WBC count, amylase, and $\mathrm{PH}$ of $(80 \%, 44 \%$, and $68 \%) ;(50 \%, 71 \%$, and $58 \%)$; and $(31 \%, 85 \%$, and $63 \%)$; respectively [12].

D-dimmer has a very high \& early sensitivity (96-100\%) 
but low specificity for diagnosing AMI in multiple studies [16].

In our study, the sensitivity of D-Dimmer was $76.9 \%$, specificity was $57.8 \%$, diagnostic accuracy was $68.8 \%$, These results match those reported by Cudnik et al. 2013 who stated that D-dimer is a promising marker for AMI with a sensitivity of $96 \%$ and a specificity of $40 \%$. [17].

The researchers try to identify new biomarkers that can recognize the early microvascular changes associated with the splanchnic \& mesenteric hypo-perfusion to allow rapid early diagnosis and prompt management that could markedly decrease the overall intestinal ischemia and reperfusion injury in addition to improving the clinical outcomes. Recently, alpha-glutathione S transferase ( $\alpha$-GST), intestinal fatty acid binding protein (I-FABP) and D-lactate are the most promising biomarkers for AMI [18].

High serum lactate is caused by release of D-lactate into the bloodstream in the late stages of intestinal ischemia \& transmural bowel infarction by anaerobic metabolism \& bacterial translocation. So, it can't detect the early phase of AMI where there is only damage to the intestinal mucosa [19].

Late intestinal ischemia \& transmural infarction may cause bowel perforation, sepsis and death. Early detection of intestinal mucosal damage by ischemia is essential because it mandates emergency surgical \& vascular interventions [9]. GST is highly active in the gut \& the liver where it is involved in detoxification of multiple toxic compounds within their cell by conjugation to glutathione. It is predominant in the cells of the intestinal mucosa which is the site of initial intestinal injury in the setting of AMI \& other stressful stimuli. $\alpha$-GST is also an indicator ischemiareperfusion injury and a predictor for antioxidant therapy in patients with intestinal ischemia [15].

In our study, $\alpha$-GST was elevated in 46 patients $(88.5 \%)$ $\&$ was normal in 6 patients $(11.5 \%)$ of the ischemic group ranging from $(0.9-45.5 \mathrm{ng} / \mathrm{ml})$ with a mean $(14.96 \mathrm{ng} / \mathrm{ml})$. The lack of increased plasma $\alpha$-GST in this patient may be related to the failure of necrotic tissue to release antioxidants as explained by Hoensch et al, 2002 [20].

In the non-ischemic group, $\alpha$-GST increased only in 8 patients $(21.1 \%)$ and was normal in 30 patients $(78.9 \%)$ with a range from 0.6 to $12.1 \mathrm{ng} / \mathrm{ml}$ and a mean $2.5 \mathrm{ng} / \mathrm{ml}$.

In our study, the specificity of $\alpha$-GST was $78.9 \%$, sensitivity $88.4 \%$, diagnostic accuracy $84.4 \%$, PPV $85.1 \%$ and NPV $83.3 \%$.

These results cope with those of Cudnik et al. 2013 who reported that in the setting of AMI, $\alpha-$ GST had a sensitivity of $68 \%$ and specificity of $85 \%$ [17].

On the contrary, Block et al, 2008 found that $\alpha$-GST had specificity $87 \%$, a sensitivity $20 \%$ and diagnostic accuracy $77.4 \%$ \& concluded that $\alpha$-GST isn't a useful or recommended biomarker [16].

Considerable limitations of $\alpha$-GST as a biomarker in AMI is that hypotension, multi-organ failure with hepatic ischemia markedly increases the plasma levels of $\alpha$-GST, serum levels of AST and ALT [4].

\section{Conclusion}

The present study evaluated \& confirmed the better diagnostic accuracy of plasma $\alpha$-GST over the classic laboratory biomarkers (e.g. lactate, amylase, CRP, WBC \& $\mathrm{PH})$ in the context of AMI. Measurement of plasma $\alpha-\mathrm{GST}$ is a simple, early, easy, and effective procedure making it a useful plasma biomarker for early diagnosis of AMI. Its high plasma levels in patients with non-specific clinical abdominal features may alert the clinician to the presence of intestinal ischemia leading to prompt early diagnosis \& expected better morbidity \& mortality outcomes. The high negative predictive value for this test in combination with other biomarkers may make it a useful screening test for AMI. However, large multi-center prospective studies are warranted to confirm these results.

\section{References}

[1] Sise MJ. Acute mesenteric ischemia. Surg Clin North Am. 2014; 94:165-81.

[2] Demir IE, Ceyhan GO, Friess H. Beyond lactate: is there a role for serum lactate measurement in diagnosing acute mesenteric ischemia? Dig Surg. 2012; 29:226-35.

[3] Hagspiel KD, Flors L, Hanley M, et al. Computed tomography angiography and magnetic resonance angiography imaging of the mesenteric vasculature. Tech Vasc Interv Radiol. 2015; 18(1):2-13.

[4] Van den Heijkant TC, Aerts BA, Teijink JA, et al. Challenges in diagnosing mesenteric ischemia. World $\mathrm{J}$ Gastroenterol. 2013; 19:1338-41.

[5] Yusuf Tanrıkulu, Ceren Şen Tanrıkulu, Mehmet Zafer et al. Diagnostic utility of the neutrophil-lymphocyte ratio in patients with acute mesenteric ischemia: A retrospective cohort study. Ulus Travma Acil Cerrahi Derg. 2016; 22(4):344-9.

[6] Whalen R, Boyer TD. Human Glutathione S Transferases. Semin Liver Dis 1998; 18(4):345-58.

[7] Delaney CP1, O'Neill S, Manning F, et al. Plasma concentrations of glutathione S-transferase isoenzyme are raised in patients with intestinal ischaemia. Br J Surg. 1999; 86(10):1349-53.

[8] Clair DG, Beach JM. Mesenteric ischemia. N Engl J Med. 2016; 374:959-68.

[9] Derikx J. P. M., Dirk Schellekens, Stefan Acosta. Serological markers for human intestinal ischemia: A systematic Review. Best Practice \& Research Clinical Gastroenterology. 2017; 31 : 69-74

[10] Wiesner W, Hauser A, Steinbrich W. Accuracy of multidetector row computed tomography for the diagnosis of acute bowel ischemia in a non-selected study population. Eur Radiol. 2004; 14:2347-56.

[11] Tilsed JV, Casamassima A, Kurihara H, et al. ESTES guidelines: acute mesenteric ischaemia. Eur J Trauma Emerg Surg, 2016; 42: 253-270. 
[12] Gearhart S, Delaney C, Senagore A et al. Prospective assessment of the predictive value of alpha-glutathione Stransferase for intestinal ischemia. Am Surg. 2003; 69(4):324-9.

[13] Duran M, Grabitz K, Schelzig H et al. The importance of open emergency surgery in the treatment of acute mesenteric ischemia. World journal of emergency surgery, 2015; 10: 45.

[14] Shuofei Yang, Xinxin Fan, Weiwei Ding, et al. D-Dimer as an Early Marker of Severity in Patients with Acute Superior Mesenteric Venous Thrombosis. Medicine 2014; 93 (29): 270.

[15] Acosta S and Nilsson T. Current status on plasma biomarkers for acute mesenteric ischemia. J Thromb Thrombolysis. 2012; 33: $355-61$.

[16] Block T., Nilsson T. K., Acosta S. Diagnostic accuracy of plasma biomarkers for intestinal ischaemia. The Scandinavian Journal of Clinical \& Laboratory Investigation. 2008; 3(68): 242-248.
[17] Cudnik MT, Darbha S, Jones J, et al. The diagnosis of acute mesenteric ischemia: a systematic review and meta-analysis. Acad Emerg Med. 2013; 20: 1087-1100.

[18] Powell A and Armstrong P. Plasma biomarkers for early diagnosis of acute intestinal ischemia. Semin Vasc Surg. 2014; 27(3-4):170-5.

[19] Björnsson S, Resch T and Acosta S. Symptomatic mesenteric atherosclerotic disease - lessons learned from the diagnostic workup. J Gastrointest Surg. 2013; 17: 973-980.

[20] Hoensch H, Morgenstern I, Petereit G, et al. Influence of clinical factors, diet, and drugs on the human upper gastrointestinal glutathione system. Gut 2002; 50:235-40. 